\title{
Evaluation of two sets of immunohistochemical and Western blot confirmatory methods in the detection of typical and atypical BSE cases
}

\author{
Chiara Porcario ${ }^{1}$, S Mark Hall $^{2 *}$, Francesca Martucci ${ }^{1}$, Cristiano Corona ${ }^{1}$, Barbara Iulini ${ }^{1}$, Alice Z Perazzini ${ }^{1}$, \\ Pierluigi Acutis ${ }^{1}$, Amir N Hamir ${ }^{3,4}$, Christina M Loiacono ${ }^{2}$, Justin J Greenlee ${ }^{3}$, Jürgen A Richt ${ }^{3,5}$, Maria Caramelli ${ }^{1}$ \\ and Cristina Casalone ${ }^{1 *}$
}

\begin{abstract}
Background: Three distinct forms of bovine spongiform encephalopathy (BSE), defined as classical (C-), low (L-) or high ( $\mathrm{H}-$ ) type, have been detected through ongoing active and passive surveillance systems for the disease. The aim of the present study was to compare the ability of two sets of immunohistochemical $(\mathrm{IHC})$ and Western blot (WB) BSE confirmatory protocols to detect C- and atypical (L- and H-type) BSE forms.

Obex samples from cases of United States and Italian C-type BSE, a U.S. H-type and an Italian L-type BSE case were tested in parallel using the two IHC sets and WB methods.

Results: The two IHC techniques proved equivalent in identifying and differentiating between C-type, L-type and $\mathrm{H}$-type BSE. The IHC protocols appeared consistent in the identification of PrPSC distribution and deposition patterns in relation to the BSE type examined. Both IHC methods evidenced three distinct PrPSC phenotypes for each type of BSE: prevailing granular and linear tracts pattern in the C-type; intraglial and intraneuronal deposits in the H-type; plaques in the L-type.

Also, the two techniques gave comparable results for PrPS ${ }^{S c}$ staining intensity on the C- and L-type BSE samples, whereas a higher amount of intraglial and intraneuronal PrPSc deposition on the H-type BSE case was revealed by the method based on a stronger demasking step.

Both WB methods were consistent in identifying classical and atypical BSE forms and in differentiating the specific $\mathrm{PrP}^{\mathrm{SC}}$ molecular weight and glycoform ratios of each form.

Conclusions: The study showed that the IHC and WB BSE confirmatory methods were equally able to recognize C-, Land H-type BSE forms and to discriminate between their different immunohistochemical and molecular phenotypes. Of note is that for the first time one of the two sets of BSE confirmatory protocols proved effective in identifying the L-type BSE form. This finding helps to validate the suitability of the BSE confirmatory tests for BSE surveillance currently in place.
\end{abstract}

\section{Background}

Transmissible spongiform encephalopathies (TSEs), or prion diseases, are fatal neurological disorders in both animals and humans. TSEs include bovine spongiform encephalopathy (BSE) in cattle, scrapie in sheep and

\footnotetext{
* Correspondence: Mark.Hall@aphis.usda.gov; cristina.casalone@izsto.it ${ }^{1}$ CEA, Istituto Zooprofilattico Sperimentale del Piemonte, Liguria e Valle d'Aosta, Via Bologna 148, 10154, Turin, Italy

${ }^{2}$ United States Department of Agriculture, Animal and Plant Health Inspection Service (APHIS), National Veterinary Services Laboratories (NVSL),

Pathobiology Laboratory, 1920 Dayton Ave, Ames, IA, 50010, USA

Full list of author information is available at the end of the article
}

goats, transmissible mink encephalopathy (TME) in mink and Creutzfeldt-Jakob disease (CJD) in humans. The agent of TSEs has been ascribed to an infectious protease-resistant isoform of the normal host encoded cellular prion protein $\left(\operatorname{PrP}^{\mathrm{C}}\right)$, termed pathological prion protein $\left(\mathrm{PrP}^{\mathrm{Sc}}\right)[1]$.

BSE was first detected in the United Kingdom (UK) in 1986 [2], where the disease grew to epidemic proportions in the cattle population, with approximately 184,600 cases diagnosed [3]. An estimated 3 million cattle may have been infected. Worldwide, about 7,900 BSE 
cases have been reported outside the UK, including Canada, Japan and the United States (U.S.) [4].

Passive and active surveillance programs were developed to monitor cattle populations for the presence of BSE. Under passive surveillance, cattle showing clinical symptoms consistent with BSE must be tested for the disease. Active surveillance programs require BSE testing by rapid screening tests at the abattoir of apparently healthy cattle of a certain age and of fallen/dead stock.

A major public health concern due to its transmissibility to humans, BSE is thought to be the cause of the fatal human prion disorder variant Creutzfeldt-Jakob disease (vCJD) [5,6]. Besides transmission among cattle and to humans, BSE can cross the species barrier to other species such as small ruminants $[7,8]$, exotic ungulates [9], and felids [10].

The origin of BSE remains an enigma: one hypothesis is that the disease was acquired by the cattle population through the consumption of meat and bone meal (MBM) derived from scrapie-affected sheep and goats or from BSE-infected cattle $[11,12]$. The efficiency of MBM feeding bans, which subsequently led to a worldwide decline in BSE among the cattle population, supports a food related spread of the disease.

Another hypothesis is that BSE may have arisen as a sporadic $[13,14]$ or genetic $[15,16]$ disease of cattle, which was then amplified by food-borne recycling of MBM containing central nervous system (CNS) tissues from BSE-infected cattle.

It was once thought that BSE was the result of a single prion strain. The disease presented with an identical clinical and pathological phenotype in cattle [17], with homogenous and consistent $\operatorname{PrP}^{\mathrm{Sc}}$ molecular features and deposition patterns in the brainstem, independently of the geographical origin of the BSE isolates detected [18-21] or breed or route of inoculation in cases of experimental transmission [22]. In transmission studies using mouse models, the BSE strain was characterized by similar incubation time, biochemical features, lesion profile and $\operatorname{PrP}^{\mathrm{Sc}}$ distribution regardless of origin $[23,24]$. But as diagnostic techniques were further refined and the BSE epidemic continued to decline, "atypical" cases of the disease, mainly in older cattle, began to be reported from around the world. The new variants were classified as low (L-) or high (H-) type based on differences in the molecular size of the $\operatorname{PrP}^{\mathrm{Sc}}$ unglycosylated protein band visualized on Western blot (WB) assays as compared to classical BSE (C-type BSE) [21].

Research into more detailed characterization of $\mathrm{PrP}^{\mathrm{Sc}}$ deposition throughout the brain of BSE-affected cattle uncovered the first example of atypical L-type BSE in Italy in 2004 [25]. Two older animals were found to be affected by a variant form of BSE, classified as L-type, based on WB analysis demonstrating faster electrophoretic mobility of $\mathrm{PrP}^{\mathrm{Sc}}$ unglycosylated moiety. WB analysis also revealed a dominant monoglycosylated $\mathrm{PrP}^{\mathrm{Sc}}$ glycoform profile for these two atypical cases. Brain samples from three additional Italian BSE-affected cattle were noted to display the same molecular features and were subsequently confirmed as atypical L-type cases.

Immunohistochemical analysis of brain sections revealed differences in the distribution and features of $\mathrm{PrP}^{\mathrm{Sc}}$ immunoreactivity between the C-type BSE and the Italian L-type BSE cases. Specifically, in three Italian Ltype BSE cases out of the five described so far, $\operatorname{PrP}^{\mathrm{Sc}}$ was found to be more abundant in the forebrain than in the brainstem where $\mathrm{PrP}^{\mathrm{Sc}}$ deposition was predominant in the C-type BSE cases. Unexpectedly, $\operatorname{PrP}^{\mathrm{Sc}}$ deposition in three out of these five Italian L-type BSE cases occurred in an unusual pattern referred to as amyloid plaques, which is seldom reported in the literature for C-type BSE [26]. Availability of only the brainstem area for the fourth Italian L-type BSE case precluded full characterization for $\mathrm{PrP}^{\mathrm{Sc}}$ plaques. At the time of this writing, the fifth L-type BSE case was undergoing characterization studies for $\operatorname{PrP}^{\mathrm{Sc}}$ plaque deposits.

On the basis of the unique neuropathological feature of $\mathrm{PrP}^{\mathrm{Sc}}$ amyloid plaques, the new L-type variant was named bovine amyloidotic spongiform encephalopathy (BASE).

The discovery of the Italian case occurred concomitantly with the detection of a second atypical BSE variant, the H-type, reported in France in 2004 [27]. Htype cases were characterized by a higher molecular weight of the unglycosylated fraction of $\operatorname{PrP}^{\mathrm{Sc}}$ than $\mathrm{C}$ type BSE and by strong labelling with P4 [28] and 12B2 [29] monoclonal antibodies. Both antibodies are directed to an $\mathrm{N}$-terminal epitope that is still present after proteinase $\mathrm{K}(\mathrm{PK})$ cleavage of $\mathrm{H}$-type BSE and also scrapie, but not of C-type or L-type BSE-derived $\operatorname{PrP}^{\mathrm{Sc}}$.

Immunohistochemical studies on the brainstem from cases confirmed as H-type from Germany [30] and the U.S. $[16,31]$ showed that the prevalent $\operatorname{PrP}^{\mathrm{Sc}}$ deposition pattern was less intense, intraneuronal and intraglial, unlike that of C-type BSE, which is more intense, with mainly granular and linear tract deposition patterns. At present, approximately 61 atypical BSE cases have been detected worldwide [as of March 2011].

In Italy, where both active and passive surveillance systems for BSE are in place, five out of 145 BSE cases detected to date were identified as BASE (L-type) variants. No H-type cases have been detected in Italy so far. However, the IHC and WB BSE confirmatory protocols in place in Italy (hereafter referred to as horse radish peroxidise [HRP] and whole homogenate extraction [WHE] methods) have proved successful in identifying 
$\mathrm{PrP}^{\mathrm{Sc}}$ deposition in H-type BSE cases from Germany [30] and other European countries, respectively [21].

In non-European countries, and the U.S. in particular, current BSE surveillance programs focus on "high-risk cattle" defined as cattle displaying clinical symptoms consistent with BSE or fallen stock. U.S. surveillance has detected three BSE cases: one C-type and two H-type BSE have been identified [31]. The efficacy of the U.S. IHC and WB BSE confirmatory protocols (hereafter referred to as alkaline phosphatase [AP] and scrapie associated fibrils extraction [SAFE] methods, respectively) to identify L-type BSE forms has not yet been tested. The purpose of this study was to evaluate Italian and U.S. IHC and WB BSE confirmatory protocols in order to assess their suitability to detect C-, H- and Ltype BSE cases. Tissue samples of classical and atypical BSE cases from Italy and the U.S., age-matched when possible, were tested by both the IHC or the WB methods used in each of the two countries.

\section{Results}

Immunohistochemical examination

\section{Description of the PrP ${ }^{S C}$ deposition patterns}

Nine different $\operatorname{PrP}^{\mathrm{Sc}}$ deposition patterns for the classical and atypical BSE cases were identified. The $\operatorname{PrP}^{\mathrm{Sc}}$ deposition types, as described elsewhere [32,20], were:

- punctate type consisting of powdery and diffuse $\operatorname{PrP}^{\mathrm{Sc}}$ staining throughout the neuropil;

- granular type characterized by granular $\mathrm{PrP}^{\mathrm{Sc}}$ accumulations in the neuropil;

- coalescing type seemingly arising from the merging of granular $\mathrm{PrP}^{\mathrm{Sc}}$ deposits to form amorphous or meshlike masses;

- glial type with $\mathrm{PrP}^{\mathrm{Sc}}$ deposits branching out from the nucleus of a glial cell on its processes, conferring it a stellate appearance;

- intraneuronal type with fine punctate $\mathrm{PrP}^{\mathrm{Sc}} \mathrm{immu}-$ noreactivity throughout the neuronal cytoplasm;

- perineuronal type consisting in thread-like $\operatorname{PrP}^{\mathrm{Sc}}$ deposits around individual neuronal perikarya and neurites;

- intraglial type with fine, punctate $\mathrm{PrP}^{\mathrm{Sc}}$ immunoreactivity adjacent to the glial nuclei;

- linear tract characterized by $\operatorname{PrP}^{\mathrm{Sc}}$ deposits along neuronal processes;

- plaques consisting of dense, generally rounded accumulations of $\operatorname{PrP}^{\mathrm{Sc}}$, unicentric or occasionally multicentric, that may present a pale core surrounded by a dark border.

\section{Comparative analysis of $\mathrm{PrP}^{S c}$ deposition using AP and HRP} methods

C-type BSE The Italian C-type BSE (16193/02) obex tissue section stained by the AP method resulted in an overall marked intensity grade for $\mathrm{PrP}^{\mathrm{Sc}}$. Moderate-to- marked punctate and granular, as well as intraneuronal and perineuronal type $\operatorname{Pr} \mathrm{P}^{\mathrm{Sc}}$ deposition patterns were evident in the hypoglossal nucleus, dorsal motor nucleus of the vagus nerve (DMNV) (Figure 1A), nucleus of the solitary tract (NST), nucleus of the spinal tract of the trigeminal nerve (NSTV), reticular formation and olivary nucleus. Mild-to-marked coalescing and linear tract type $\mathrm{PrP}^{\mathrm{Sc}}$ deposition was present in the reticular formation. In serial sections of the obex very similar results were obtained with the HRP method, with no detectable differences in $\mathrm{PrP}^{\mathrm{Sc}}$ distribution, deposition pattern or staining intensity (Figure 1B).

The U.S. C-type BSE case (B5330-7 D) examined under the AP protocol contained overall moderate-tomarked $\mathrm{PrP}^{\mathrm{Sc}}$ immunoreactivity. There was moderate punctate, granular, intraneural and perineuronal type $\mathrm{PrP}^{\mathrm{Sc}}$ deposition in all the nuclei of the obex (Figure $1 C)$. Glial, granular and linear tract $\mathrm{PrP}^{\mathrm{Sc}}$ deposition patterns were mainly detected in the reticular formation.

The HRP protocol applied to serial sections of the U. S. C-type BSE case stained with the same neuroanatomical $\mathrm{PrP}^{\mathrm{Sc}}$ distribution as seen under the AP protocol. Furthermore, $\operatorname{PrP}^{\mathrm{Sc}}$ deposition patterns and related intensity for all the target nuclei appeared similar to the corresponding obex sections stained under the AP protocol (Figure 1D).

H-type BSE The H-type BSE case (B14842) showed overall moderate $\operatorname{PrP}^{\mathrm{Sc}}$ immunoreactivity when stained

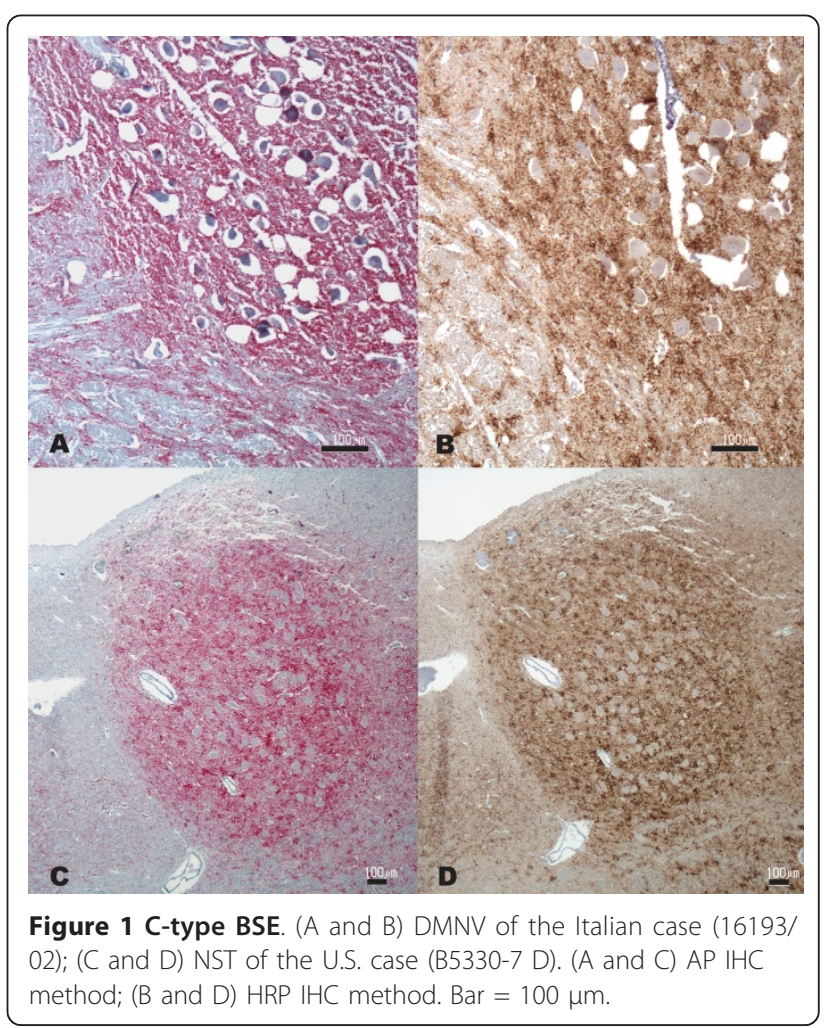


under the AP protocol. $\mathrm{PrP}^{\mathrm{Sc}}$ was mainly detected at the level of the DMNV, NST, NSTV and the reticular formation, primarily appearing as punctate and granular deposits with areas of intraglial deposition (Figure 2A). Intraneuronal and perineuronal $\mathrm{PrP}^{\mathrm{Sc}}$ deposition was present in the DMNV and the reticular formation (Figure $2 \mathrm{C}$ ).

Serial tissue sections of the same case stained under the HRP protocol revealed more widespread $\mathrm{PrP}^{\mathrm{Sc}}$ distribution and moderate-to-marked immunolabelling intensity. $\mathrm{PrP}^{\mathrm{Sc}}$ was detected through the whole tissue section. Interestingly, the HRP method revealed more intraglial (Figure 2B) and intraneuronal (Figure 2D) deposition patterns, with a higher degree of staining intensity than the AP method.

L-type BSE The L-type BSE (BASE) (12966/07) obex tissue sections examined under the AP protocol contained mild-to-moderate $\mathrm{PrP}^{\mathrm{Sc}}$ immunoreactivity. The prevalent $\operatorname{PrP}^{\mathrm{Sc}}$ deposition pattern was the punctate and granular type, which was mildly present in the hypoglossal and olivary nucleus and moderately present at the level of the DMNV, NST, NSTV, and reticular formation. Intraneuronal type $\operatorname{PrP}^{\mathrm{Sc}}$ deposition was identified in some neurons of the hypoglossal nucleus, DMNV, NSTV, reticular formation and olivary nuclei.

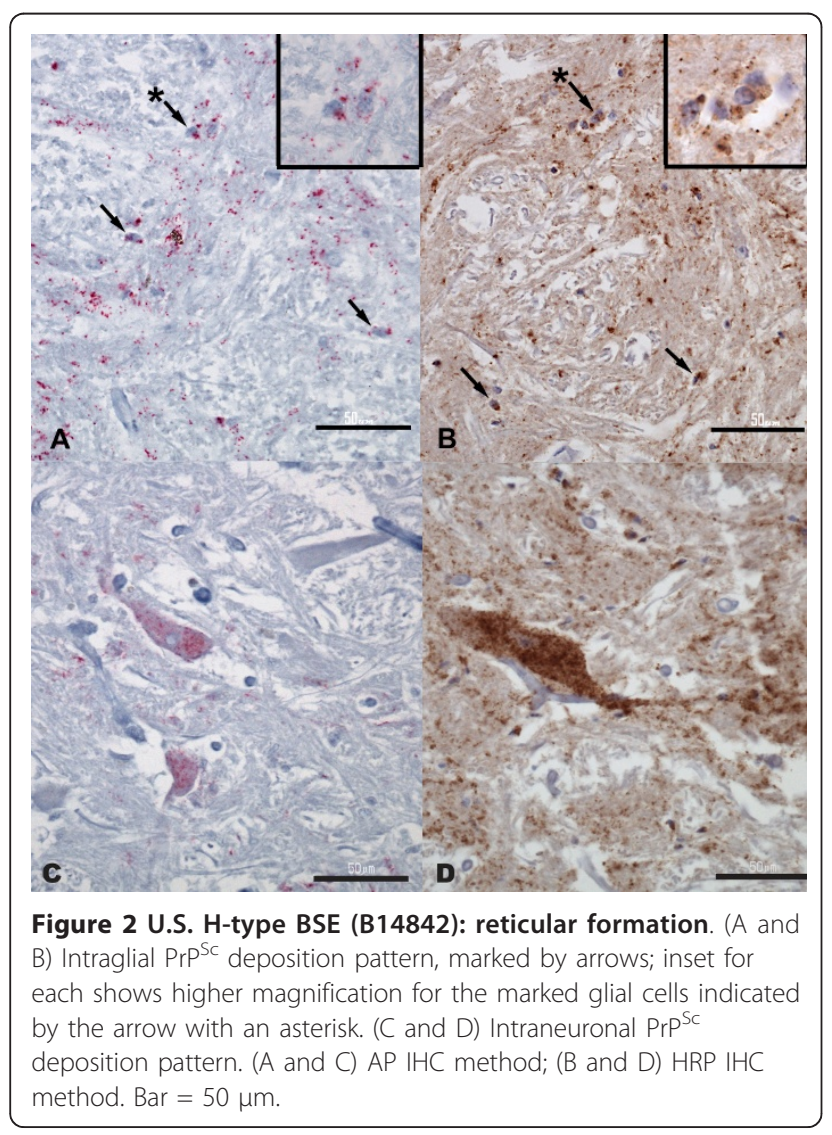

Perineuronal type $\mathrm{PrP}^{\mathrm{Sc}}$ immunoreactivity of moderate intensity was identified in the DMNV (Figure 3A), reticular formation and the olivary nuclei. Linear tract type $\mathrm{PrP}^{\mathrm{Sc}}$ deposits were present at the level of the reticular formation. Also present in the reticular formation were several larger aggregates of staining or plaques ranging from 5 to $25 \mu \mathrm{m}$ in diameter. The majority of the plaques appeared as unicentric structures, but some were characterized by the presence of a pale core surrounded by a dark radial periphery.

The results obtained on the serial obex tissue sections of the BASE case stained according to the HRP method were comparable in terms of $\operatorname{PrP}^{\mathrm{Sc}}$ distribution, staining intensity and deposition pattern (Figure 3B).

The frontal cortex of the BASE case stained under the AP protocol contained moderate punctate, granular type $\mathrm{PrP}^{\mathrm{Sc}}$ deposition along the most superficial region of the cortex. In the deeper regions of the gray matter, a mild granular $\operatorname{PrP}^{\mathrm{Sc}}$ deposition pattern was present. A similar pattern with moderate staining intensity was present along the transition zone between the gray and white matter. In the white matter there were granular $\operatorname{PrP}^{\mathrm{Sc}}$ deposits and scattered $\operatorname{PrP}^{\mathrm{Sc}}$ positive plaques that appeared as dense unicentric or multicentric, dark red, round structures (Figure $3 \mathrm{C}$ ).

Serial tissue sections of the frontal cortex from this animal stained using the HRP procedure revealed a similar distribution of $\operatorname{PrP}^{\mathrm{Sc}}$ deposits, including the $\mathrm{PrP}^{\mathrm{Sc}}$ pattern types described above and comparable immunoreactivity intensity. The plaques appeared as

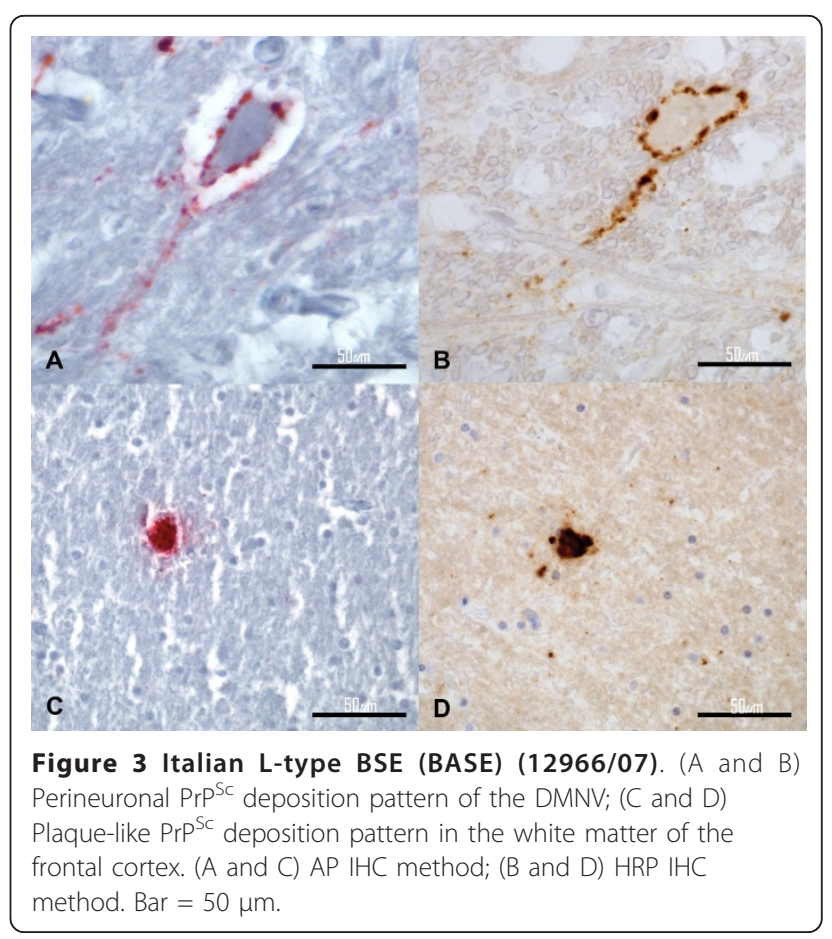


dense, unicentric, dark brown, round structures, some of which displaying a pale core surrounded by a dark radial periphery (Figure 3D).

\section{Western blot analysis}

The obex tissue derived from a healthy bovine and used as control for PK digestion tested negative with both extraction methods. The WHE and SAFE extraction methods were both able to clearly identify classical and atypical BSE cases on subsequent WB analyses. When the $6 \mathrm{H} 4 \mathrm{mAb}$ was employed, which is directed against the protease-resistant core of the prion protein, all BSE samples (C-, H-, L-type BSE cases) tested positive (Figure 4A). In contrast, when the P4 mAb was used, which is directed against the $\mathrm{N}$-terminus of the protease-resistant core of PrP, only the $\mathrm{H}$-type BSE sample was recognized (Figure 4B) by both extraction methods. The density comparison between the signals obtained with $\mathrm{P} 4$ and $6 \mathrm{H} 4 \mathrm{mAbs}$ showed a $\mathrm{P} 4 / 6 \mathrm{H} 4$ ratio of at least 1.2 for both methods in the H-type BSE sample. These findings were due to the different PK cleavage site for the three BSE types, as previously described by Jacobs et al. (21).

In addition, the glycoform ratio of the different BSE samples was determined after staining with the $6 \mathrm{H} 4$ mAb. As shown in Figure 5, the L- and H-type BSE cases showed a lower proportion of the di-glycosylated and a higher proportion of the mono-glycosylated band than the C-type BSE cases according to both extraction methods. Specifically, the L-type BSE exhibited an equal or slightly inverted ratio between the mono- and the di-

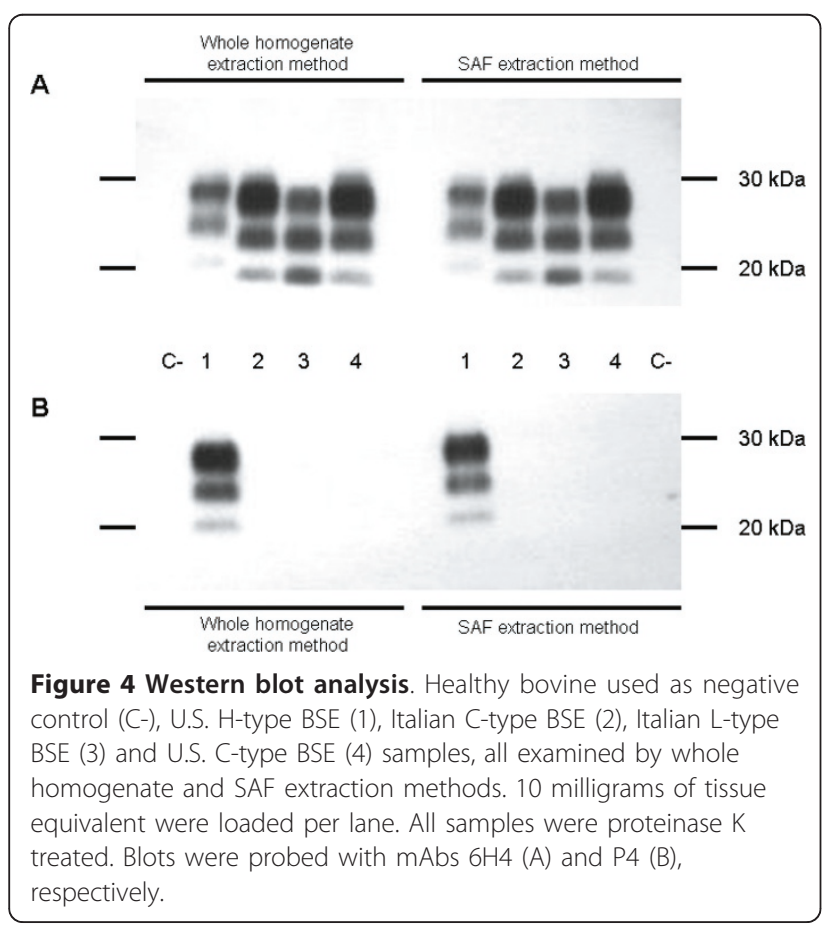

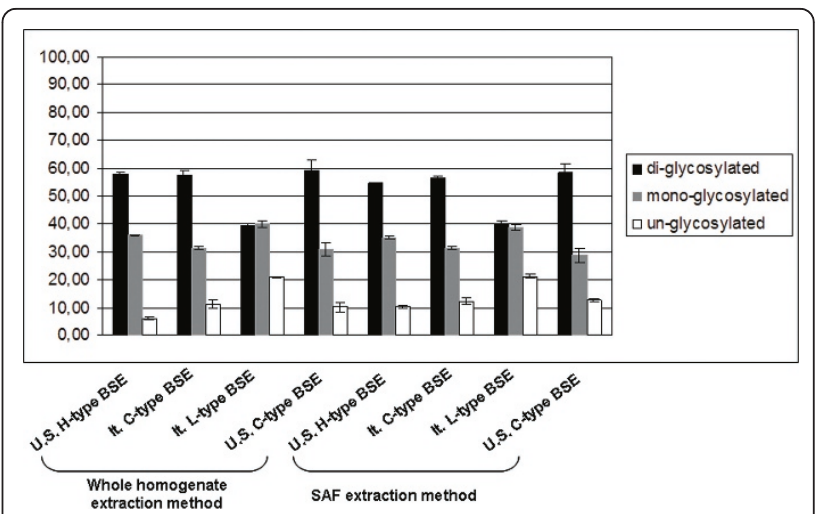

Figure 5 Glycoform analysis. Graphic representation of the proportions of the three PrPs ${ }^{\mathrm{Sc}}$ glycoforms of the samples examined by the whole homogenate and the SAF extraction methods.

glycosylated PrP isoform, which is a characteristic feature of L-type BSE (Figure 5).

\section{Discussion and conclusions}

The aim of this study was to evaluate the suitability of IHC and WB confirmatory tests routinely used in the U. S. and Italy for the diagnosis of classical and atypical BSE. In contrast to C-type BSE cases, which have progressively declined worldwide after the implementation of feed bans, atypical BSE (L- and H-type BSE) cases have been increasingly detected since 2004, and are postulated to arise as sporadic and genetic forms of the disease [14-16]. Most likely, the incidence of atypical BSE cases will be constant over time [14], as occurs in sporadic and genetic cases of human prion diseases [33]. The existence of atypical forms of BSE will require maintaining the current active surveillance systems for BSE; the misguided proposal many countries have put forward to progressively limit them could result in such cases being missed. Nonetheless, the feed bans on specified risk materials will continue to provide effective barriers for protecting public health.

All atypical BSE cases detected worldwide so far have been correctly identified by currently approved BSE rapid tests $[34,35]$. However, it should be noted that Ltype BSE (BASE), the only BSE variant phenotypically characterized in detail to date [25], may contain significantly less $\mathrm{PrP}^{\mathrm{Sc}}$ immunoreactivity in the obex region than C-type BSE. Since the obex is the target tissue for the diagnosis of all BSEs, it is necessary to evaluate the sensitivity of rapid testing and confirmatory diagnostic techniques currently in place for atypical forms. In the future, it may be necessary to re-evaluate whether the obex is the best tissue for detecting atypical BSE cases.

One aim of the present study was to assess the effectiveness of the U.S. BSE confirmatory methods to detect L-type BSE. The results indicate that the AP and SAFE 
BSE confirmatory protocols are fully adequate to identify the presence of $\mathrm{PrP}^{\mathrm{Sc}}$ in the L-type atypical BSE forms. The efficacy of the AP and SAFE confirmatory methods to detect $\mathrm{C}$ - and $\mathrm{H}$-type BSE has already been shown [16,31].

When the AP protocol was applied to obex and frontal cortex tissue sections of an Italian L-type BSE (BASE) case, $\mathrm{PrP}^{\mathrm{Sc}}$ was detected with the same neuroanatomical distribution, deposition pattern and staining intensity as the HRP method. More specifically, the AP method revealed, at both the obex and frontal cortex, a characteristic neuropathologic trait of the Italian L-type $\mathrm{BSE}$ form, i.e., the presence of $\operatorname{PrP}^{\mathrm{Sc}}$ aggregates in the form of plaques. Another peculiar feature described in cattle affected by natural [30] and experimental [36] Ltype BSE is the presence of a perineuronal $\operatorname{PrP}^{\mathrm{Sc}}$ deposition pattern which is not associated with intraneuronal $\mathrm{PrP}^{\mathrm{Sc}}$ deposits. This $\mathrm{PrP}^{\mathrm{Sc}}$ pattern was also detected by both the HRP and AP methods in the Italian L-type BSE case in this study. It has been hypothesized that the perineuronal $\mathrm{PrP}^{\mathrm{Sc}}$ deposits could indicate different $\mathrm{PrP}^{\mathrm{Sc}}$ trafficking and propagation in L-type BSE forms [36].

This comparative study also confirmed the reliability of the HRP and WHE confirmatory protocols in the diagnosis of the $\mathrm{H}$-type BSE form not reported in Italy to date. Previous investigations performed on $\mathrm{H}$-type samples obtained from other European countries revealed that the HRP technique was successful in detecting H-type BSE cases [30].

Under both the HRP and AP protocols, the U.S. Htype BSE case displayed a similar $\mathrm{PrP}^{\mathrm{Sc}}$ deposition pattern of the granular, intraneuronal, and intraglial type. At present, only the obex and not a complete brain from an $\mathrm{H}$-type BSE naturally affected cow has been available for analysis. In the obex region of H-type BSE cases, intraglial $\mathrm{PrP}^{\mathrm{Sc}}$ deposition seems to be a peculiar immunohistochemical feature, as described in cases from Germany [30] and Sweden [37]. It is a less common feature for C- and L-type BSE cases [20,25]. Importantly, the intraglial pattern identified in a Swedish $\mathrm{H}$ type BSE case was revealed only with antibodies (F99/ 97.6.1 and R145) directed against the C-terminus portion of the $\mathrm{PrP}^{\mathrm{Sc}}$ molecule [37]. In contrast, the detection of severe granular intracellular labelling in German $\mathrm{H}$-type BSE with the use of core-specific mAb 12F10 has also been reported [30].

The U.S. H-type BSE case examined here had more intense and defined $\operatorname{PrP}^{\mathrm{Sc}}$ immunoreactivity of intraneuronal and intraglial distribution when tested using the HRP as compared to the AP method. This was surprising, since the AP protocol employs a ten-fold higher concentration of the primary F99/97.6.1 antibody as compared with the one employed by the HRP protocol.
Moreover, as compared to the HRP method, the higher incubation temperature $\left(37^{\circ} \mathrm{C}\right)$ at which the AP staining protocol occurs in the Ventana NexES carousel ought to confer a more efficient binding of the primary antibody to the tissue antigens. However, such preferable conditions in the AP method could partially be negatively influenced by a possible prozone-like effect due to the higher concentration of the primary antibody employed. The formation of antibody aggregates might limit their penetration into the cells and restrict binding to intracytoplasmatic antigenic sites [38]. Furthermore, it can not be excluded that the overall shorter incubation times of the AP protocol as compared to the HRP method might reduce the antibody-antigen interaction. These assumptions aside, the discrepancy between the AP and the HRP methods in the amount of $\mathrm{PrP}^{\mathrm{Sc}}$ detected in the $\mathrm{H}$-type case seems more likely to be related to the different length of the formic acid treatment performed on tissue sections after deparaffinization.

The HRP protocol utilizes a 25-min formic acid incubation, whereas the AP protocol limits formic acid exposure to $5 \mathrm{~min}$. To perform immunohistochemical analysis on formalin-fixed tissues, a demasking step in formic acid is often used to expose antigenic sites to the binding by antibodies [39]; this step seems to be particularly critical to disclose the intracellular $\mathrm{PrP}^{\mathrm{Sc}}$ component in $\mathrm{H}$-type BSE cases. The $\operatorname{PrP}^{\mathrm{Sc}}$ molecule characterizing $\mathrm{H}$-type $\mathrm{BSE}$ probably requires prolonged exposure to formic acid to be properly demasked in formalin-fixed tissues and to be revealed, mainly by employing C-terminal antibodies, as intraneuronal and intraglial $\mathrm{PrP}^{\mathrm{Sc}}$ deposits.

By contrat, a shorter demasking time in formic acid, i. e., $5 \mathrm{~min}$ as employed in the AP protocol, does not seem to affect the detection of the $\mathrm{PrP}^{\mathrm{Sc}}$ plaque deposits, which may be a unique feature of L-type BSE cases. This finding suggests that in L-type BSE isolates the length of incubation time in formic acid is not a very critical step to disclose $\mathrm{PrP}^{\mathrm{Sc}}$ deposits, perhaps indicating that L-type $\operatorname{PrP}^{\mathrm{Sc}}$ most likely establishes weaker interactions with formalin and can therefore be more easily demasked than $\mathrm{H}$-type $\mathrm{PrP}^{\mathrm{Sc}}$ from formalin-fixed, paraffin-embedded tissue sections.

In summary, we found that comparative testing of an automated immunostaining protocol, with the use of the Ventana NexES Autostainer System, and a manual IHC method in the detection of $\operatorname{PrP}^{\mathrm{Sc}}$ in various BSE cases revealed absolute concordance in correctly identifying BSE-positive samples and in discriminating among C-, $\mathrm{H}$ - and L-type forms of BSE. Both the HRP and the AP BSE confirmatory methods differentiated three different phenotypes of the disease, referred to as C-, H- and Ltype forms, each appearing to be characterized by quite distinct features of $\operatorname{PrP}^{\mathrm{Sc}}$ deposition. Both methods 
revealed granular and linear tract $\mathrm{PrP}^{\mathrm{Sc}}$ deposits as a distinguishing feature of C-type BSE cases, whereas intraglial and intraneuronal $\operatorname{PrP}^{\mathrm{Sc}}$ deposition patterns appeared as the most representative trait of $\mathrm{H}$-type BSE, and the presence of $\mathrm{PrP}^{\mathrm{Sc}}$ deposits organized as plaques was only detected in the L-type BSE (BASE) case.

In contrast to the IHC protocols, the WHE and the SAFE procedures for WB methods are almost identical. Both are, in fact, based on the enrichment of samples according to scrapie associated fibrils (SAF) immunoblotting methods derived from the World Organization for Animal Health (OIE) recommended protocol. The minor differences between the two protocols, for example, the use of different homogenization buffers or the sonication step, do not seem to affect results in terms of specificity and sensitivity to detect BSE cases. Furthermore, the fact that under both the WHE and SAFE protocols a higher amount of tissue equivalents (i.e., $10 \mathrm{mg}$ ) than that normally used for medium positive cases (i.e., $\sim 500 \mu \mathrm{g}$ ) had to be loaded per lane is not to be ascribed to the sensitivity of the methods but simply to the need to display as best as possible the low signal intensity known to characterize the $\operatorname{PrP}^{\mathrm{Sc}}$ moieties of some of the BSE cases under examination. Previous similar attempts to maximise at $\mathrm{WB}$ analysis the $\operatorname{PrP}^{\mathrm{Sc}}$ signal intensity of slightly positive BSE samples by loading even higher amounts of tissue equivalents per lane (i.e., $38 \mathrm{mg}$ ) than the quantity employed here have also been reported (27).

Our findings show that both the SAFE and WHE methods were able to detect all three BSE forms (C-, $\mathrm{H}$ - and L-type). The WHE and SAFE methods identified and differentiated the specific molecular weights and glycoform ratios associated with the three BSE forms. As regards the H-type BSE in particular, it is noteworthy that both the SAFE and WHE methods clearly detected this atypical form, even when an antibody such as P4 was employed, which is known to be inferior for application to bovine PrP compared to other antibodies such as $12 \mathrm{~B} 2$ (29) or $6 \mathrm{H} 4$. The results provide reliable evidence that the SAFE method can recognize and differentiate L-type BSE in the current surveillance program.

The present study proved that both Italy and the U.S. employ efficient diagnostic techniques to detect and differentiate the three different BSE forms (C-, $\mathrm{H}$ - and Ltype) known to date. Furthermore, this finding suggests that the current Italian and U.S. confirmatory methods for BSE might also be appropriate to disclose novel forms of the disease should they ever occur.

\section{Methods}

\section{U.S. tissues}

The only C-type BSE case detected in the U.S. to date, identified as animal B5330-7 D, was examined. This animal was an approximately 6.5-year-old, as determined by dentition, non-ambulatory Holstein Friesian cow, imported from Canada in 2001 and slaughtered in Moses Lake, WA in 2003 [31] (Table 1). In accordance with the BSE surveillance system in place at that time, the case underwent confirmatory diagnosis by means of histology and IHC.

Two H-type BSE cases, both U.S. born and identified as animals B10979 and B14842, were examined. Case B10979 was an approximately 12-year-old Brahman cross cow that died during transport to a packing plant [31] (Table 1). The case was first detected by rapid testing (Platelia/TeSeETM ELISA BSE test, Bio-Rad, Hercules, CA) and later confirmed positive by WB and IHC analysis in the U.S. and at the OIE Reference Laboratory for BSE in Weybridge, England. Case B14842 was an approximately 10-year-old crossbred (Bos indicus $\times$ Bos taurus) downer cow euthanized on the owner's farm by the herd veterinarian [16]. This case was also initially detected by rapid testing (Platelia/TeSeETM ELISA BSE test, Bio-Rad, Hercules, CA), then confirmed positive by IHC and WB analysis (Table 1).

Table 1 Details of the C-, H- and L-type BSE cases examined

\begin{tabular}{|c|c|c|c|c|c|c|c|c|}
\hline Case & Country & $\begin{array}{c}\text { Age } \\
\text { (year) }\end{array}$ & Breed & Cause of death & Husbandry & BSE type & Tissue & Tested in the study by \\
\hline $16193 / 02$ & Italy & 5 & Friesian & r.s.* & dairy & C-type & O. & $\mathrm{IHC}$ \\
\hline $142585 / 03$ & Italy & 11 & Mixed & c.s.t & beef & C-type & o. & WB \\
\hline B5330-7 D & USA & $\sim 6.5$ & Friesian & c.s.t & dairy & C-type & O. & $\| \mathrm{HC}, W B$ \\
\hline B10979 & USA & $\sim 12$ & Brahma cross & f.s. $\neq$ & beef & H-type & O. & WB \\
\hline B14842 & USA & $\sim 10$ & Red crossbred & f.s. $\neq$ & beef & H-type & O. & $\mathrm{HC}$ \\
\hline 1088/03 & Italy & 15 & Piedmontese & f.s.‡ & dairy/beef & L-type & O. & WB \\
\hline 12966/07 & Italy & 14 & Piedmontese & f.s. $\neq$ & dairy/beef & L-type & o. , f.c. & $\mathrm{IHC}$ \\
\hline
\end{tabular}

* r.s., routine slaughtered

† c.s., casualty slaughtered

$\neq$ f.s., fallen stock

\# o., obex

$\S$ f.c., frontal cortex 


\section{Italian tissues}

Two C-type BSE cases were examined. Case 16193/02 was a 5-year-old routinely slaughtered Friesian cow and case 142585/03 was a slaughtered 11-year-old beef cow of mixed breed (Table 1). According to the Italian surveillance programs, both cases were first detected by a rapid screening test (Prionics Check Western, Prionics, Zurich, Switzerland) on the brainstem, and then confirmed by histology, IHC and WB analysis.

Two L-type BSE (BASE) cases, identified as animal $12966 / 07$ and animal 1088/03 [25] were examined. Both cases were fallen stock Piedmontese cows, 14 and 15 years old respectively (Table 1 ), initially detected by rapid testing and then confirmed positive by BSE confirmatory tests.

\section{Immunohistochemistry (IHC) Case selection and preparation of tissue sections}

The Italian C-type BSE case (16193/02), U.S. C-type BSE case (B5330-7 D), Italian L-type (BASE) case (12966/07) and U.S. H-type case (B14842) were examined by IHC under both the HRP and the AP protocols. Obex sections for all cases and additional sections of the frontal cortex of the L-type case were tested (Table 1).

Serial sections were cut and numbered consecutively in order to test tissue sections, alternating between the AP protocol and the HRP method. Three slides of each tissue sample selected for the study were tested under the HRP protocol: two sections were incubated with the primary antibody; one, on which the primary antibody was omitted, was used as an internal control. Two slides of each tissue sample underwent IHC according to the AP protocol, and the primary antibody was applied to both of them.

The IHC staining comparison study was performed at the National Veterinary Services Laboratories (NVSL) in Ames, IA.

\section{AP protocol}

Tissues selected for examination were fixed in $10 \%$ buffered formalin, embedded in paraffin blocks, sectioned at $5 \mu \mathrm{m}$ and mounted onto charged glass slides (Fisher Superfrost Plus, Fisher Scientific, Hampton, NH). The slides were air dried for a minimum of $3 \mathrm{~h}$, put in an oven for $15 \mathrm{~min}$ at $80^{\circ} \mathrm{C}$, deparaffinized in xylene, and rehydrated in graded alcohols, followed by distilled $\mathrm{H}_{2} \mathrm{O}$. They were then incubated in $95 \%$ formic acid for $5 \mathrm{~min}$, followed by three washes ( 2 min each) in Tris Buffer $\mathrm{pH}$ 7.5 and a 1-min wash in laboratory quality water. The slides were immersed in an antigen retrieval citrate buffer, pH 6 (DAKO ${ }^{\text {Tм}}$ Target Retrieval Solution, Dako North America Inc., Carpinteria, CA) and placed in a commercially available antigen retrieval device (Biocare Medical, Concord, CA) for $30 \mathrm{~min}$ at $121^{\circ} \mathrm{C}$. After the slides were allowed to cool to room temperature (RT), they were stained using an automated immunostainer (NexES IHC module, Ventana Medical Systems Inc., Tucson, AZ), previously filled with the PrP-specific F99/ 97.6.1 antibody (epitope QYQRES, aa 220-225 of the ovine PrP, $10 \mu \mathrm{g} / \mathrm{ml}$ dilution; VMRD Inc., Pullman, WA) [40], APK wash solution (Ventana Medical Systems Inc.) and liquid coverslip bottles (Dako North America Inc.). As suggested by the manufacturer, all incubations were performed on a Ventana NexES platform at $37^{\circ} \mathrm{C}$ following the alkaline phosphatase red paraffin protocols. The first step associated with the autostainer included incubation of sections with the F99/97.6.1 anti-PrP antibody for $32 \mathrm{~min}$, rinsing and application of coverslip solution. The following steps were performed employing reagents provided by the Alkaline Phosphatase Basic Red Kit (Ventana Medical Systems Inc.) and involved incubation of the slides with a biotinylated goat antimouse IgG for $8 \mathrm{~min}$, application of an avidin-alkaline phosphatase complex for $12 \mathrm{~min}$, followed by incubation with an Enhancer reagent for a 4 min. Naphthol was used as substrate for the reaction and applied for 8 min together with Fast Red A, used as chromogen. Incubation with Fast Red B, used as second chromogen, followed for $8 \mathrm{~min}$. The sections were subsequently counterstained with Gill's hematoxylin (Ventana Medical Systems Inc.) for $4 \mathrm{~min}$ and then postcounterstained with bluing reagent (Ventana Medical Systems Inc.) for $2 \mathrm{~min}$. At the end of the process, the slides were removed from the automated stainer and dipped in warm tap water with 2-3 drops of liquid dishwashing detergent added (Non Ultra Dish Liquid, Original Scent, Dawn. Procter \& Gamble, Cincinnati, $\mathrm{OH}$ ). After washes in tap water, the sections were dehydrated in ethyl alcohol and xylene and then coverslipped with a permanent mounting medium.

Test slides were examined by light microscopy and classified as positive or negative for $\mathrm{PrP}^{\mathrm{Sc}}$ immunoreactivity. $\mathrm{PrP}^{\mathrm{Sc}}$ immunoreactivity was defined as pink to red staining; slides identified as negative for $\mathrm{PrP}^{\mathrm{Sc}}$ contained only counterstaining which appeared blue. As positive controls, slides from the obex tissue of a scrapie positive sheep, obtained from the NVSL, and from the brainstem and the frontal cortex, respectively, of two experimentally infected TME positive cows, provided by the National Animal Disease Center (NADC), were employed. Obex tissue sections of an Italian BSE negative cow were utilized as negative controls.

\section{HRP protocol}

The Italian samples selected for the study were formalin-fixed and then treated for decontamination with $98 \%$ formic acid for $1 \mathrm{~h}$ before being embedded in paraffin blocks. Under the HRP protocol, sections were cut $5 \mu \mathrm{m}$ thick for each sample, placed onto charged glass slides, drained, air dried for $10 \mathrm{~min}$ and then incubated at $37^{\circ}$ 
C overnight. Dewaxing was performed in four 6-min steps: the first two in xylene, the next two in absolute ethyl alcohol. The slides were then rinsed and immersed in $98 \%$ formic acid for $25 \mathrm{~min}$. After washing in distilled water, the rack of slides was put into a container filled with fresh epitope demasking solution (citrate buffer $\mathrm{pH}$ 6.1) with anti-bump glass beads at the bottom. The sections were then autoclaved for $30 \mathrm{~min}$ at $121^{\circ} \mathrm{C}$. Endogenous peroxidase activity was blocked using $3 \%$ hydrogen peroxide in methanol for $20 \mathrm{~min}$ at RT. To block non-specific tissue antigens, the sections were incubated with $5 \%$ horse blocking serum for $20 \mathrm{~min}$ at $\mathrm{RT}$, then incubated for $1 \mathrm{~h}$ at $\mathrm{RT}$ with the primary monoclonal antibody (MAb) F99/97.6.1 (epitope QYQRES, aa 220-225 of the ovine PrP, $1 \mu \mathrm{g} / \mathrm{ml}$ dilution; VMRD Inc.) [40]. After rinsing, a biotinylated goat antimouse secondary antibody (1:200 dilution; Vectastain ABC kit, Vector Laboratories, Burlingame, CA) was applied to the tissue sections for $30 \mathrm{~min}$ at RT, followed by the avidin-biotin-peroxidase complex (Vectastain ABC kit, Vector Laboratories) for a minimum of 30 min. $\mathrm{PrP}^{\mathrm{Sc}}$ immunoreactivity was visualized using 3-3'diaminobenzidine (Dakocytomation, Carpinteria, CA) as chromogen; the sections were then counterstained with Meyer's hematoxylin, dehydrated in ethyl alcohol and xylene and coverslipped. $\operatorname{PrP}^{\mathrm{Sc}}$ immunoreactivity appeared from light to dark brown, whereas slides identified as negative for $\mathrm{PrP}^{\mathrm{Sc}}$ staining contained only counterstaining which appeared blue. Positive and negative tissue controls for the HRP protocol were identical to the aforementioned controls used for the AP protocol.

\section{Assessment of PrP ${ }^{S c}$ immunoreactivity}

For the C- and L-type BSE cases, the obex was sampled at the neuroanatomical level including the following nuclei: NST; DMNV; NSTV; the nucleus of the hypoglossal nerve; the olivary nucleus; the reticular formation. This allowed for the identification of $\operatorname{PrP}^{\mathrm{Sc}}$ deposition and distribution.

For the L-type BSE (BASE) case, an area of frontal cortex was also examined.

Assessment of $\mathrm{PrP}^{\mathrm{Sc}}$ immunoreactivity was based on neuroanatomical location, morphology and intensity $(0$, ,,++++++ ) where 0 denotes no $\mathrm{PrP}^{\mathrm{Sc}},+$ denotes mild intensity, ++ denotes moderate intensity, and ++ + denotes marked intensity [20]. This assessment of $\operatorname{PrP}^{\mathrm{Sc}}$ intensity is subjective.

\section{Western blot}

Frozen obex samples from the Italian C-type BSE case (142585/03), U.S. C-type BSE case (B5330-7 D), Italian L-type (BASE) case $(1088 / 03)$ and U.S. H-type case (B10979) and CNS tissue from a healthy bovine, used as negative control, were tested under both the WHE and the SAFE WB protocols (Table 1).
A portion of the Italian C- and L-type BSE cases was shipped to the Agricultural Research Service (ARS) Laboratories of the NADC, Ames, Iowa, where the WB comparison study was performed.

\section{SAFE protocol}

The OIE-recommended SAF-Immunoblot method [41] was used with minor modifications. Tissue homogenates $(10 \%[\mathrm{w} / \mathrm{v}])$ in $10 \mathrm{mM}$ Tris, $\mathrm{pH} \mathrm{7.5,} \mathrm{containing} 5 \mathrm{mM}$ $\mathrm{MgCl}_{2}$ were prepared. The homogenates were sonicated for $30 \mathrm{~s}$ in an ice bath. Benzonase nuclease $(100 \mathrm{U} / \mathrm{ml}$; Novagen, Merck KGaA, Darmstadt, Germany) was added to the homogenates and they were incubated for $1 \mathrm{~h}$ at $37^{\circ} \mathrm{C}$ while shaking. An equal volume of $20 \%$ (w/v) sarcosyl in $10 \mathrm{mM}$ Tris, $\mathrm{pH} 7.5$ and $1 \mathrm{mM}$ dithiotreithol (DTT) was added to each homogenate, vortexed for 1 min every $10 \mathrm{~min}$ for a total of $30 \mathrm{~min}$ at RT. The homogenates were transferred to polyallomer tubes and centrifuged at $20,000 \mathrm{~g}$ for $25 \mathrm{~min}$ at $10^{\circ} \mathrm{C}$. Supernatants were collected and centrifuged again at 200,000 $\mathrm{g}$ for $55 \mathrm{~min}$ at $10^{\circ} \mathrm{C}$. The resultant supernatants were discarded. The pellets were resuspended in sterile, distilled water $(1 \mu \mathrm{l} /$ mg tissue equivalent) and sonicated until suspended. The samples were treated with PK $(40 \mu \mathrm{g} / \mathrm{ml})$ by incubation at $37^{\circ} \mathrm{C}$ for $1 \mathrm{~h}$ with agitation. Phenylmethylsulphonyl fluoride (PMSF) was added to a final concentration of 5 $\mathrm{mM}$, and the samples were incubated on ice for $15 \mathrm{~min}$. The volume was brought up to $500 \mu \mathrm{l}$ with water and centrifuged at $200,000 \mathrm{~g}$ for $1 \mathrm{~h}$ at $10^{\circ} \mathrm{C}$. Supernatants were discarded and the pellets were resuspended in sodium dodecyl sulphate polyacrylamide gel electrophoresis (SDS-PAGE) sample buffer (Invitrogen, Carlsbad, CA).

\section{WHE protocol}

Samples were extracted by the WHE method previously reported [42]. Briefly, 10\% (w/v) brainstem tissues were homogenized in sarcosyl-Tris buffered saline solution, $\mathrm{pH}$ 7.5. The homogenates were clarified by centrifugation at $22,000 \mathrm{~g}$ for $20 \mathrm{~min}$ at $10^{\circ} \mathrm{C}$ and the supernatants were incubated with PK $(40 \mu \mathrm{g} / \mathrm{ml})$ for 1 $\mathrm{h}$ at $37^{\circ} \mathrm{C}$ under continuous shaking. Digestion was stopped by adding $10 \mu \mathrm{l}$ of PMSF $100 \mathrm{mM}$ to the samples. After ultracentrifugation (Ultracentrifuge Optima TLX, Rotor TLA 110, Beckman Coulter, Fullerton, CA) at $215,000 \mathrm{~g}$ for $1 \mathrm{~h}$ at $10^{\circ} \mathrm{C}$, the supernatants were discarded and pellets were resuspended in Laemmli buffer.

\section{Western blot analysis}

The samples extracted by the two methods were boiled for $10 \mathrm{~min}$ at $99^{\circ} \mathrm{C}$, then separated by SDS-PAGE on 4$12 \%$ minigels (Invitrogen) and transferred onto polyvinylidene difluoride (PVDF) membranes (Immobilon-P, Millipore, Billerica, MA). Blots were blocked with PVDF blocking buffer (Invitrogen) for $1 \mathrm{~h}$ at $\mathrm{RT}$, and blotted bovine PrP was detected with $6 \mathrm{H} 4(0.2 \mu \mathrm{g} / \mathrm{ml}$; Prionics $)$ 
[43] and P4 (0.1 $\mu \mathrm{g} / \mathrm{ml}$; R-Biopharm, Darmstadt, Germany) [28] monoclonal antibodies, respectively. After three washes with Tris buffered saline Tween 20 (TBST) (Invitrogen), the blots were incubated for $30 \mathrm{~min}$ at RT with a biotinylated sheep anti-mouse antibody (GEHealthcare Ltd., St. Giles, UK). After another round of washes, streptavidin-horseradish peroxidase conjugate (GE-Healthcare Ltd.) incubation was done. Membranes were developed using ECL Plus chemiluminescent substrate (GE-Healthcare Ltd.) onto Hyperfilm ECL (GEHealthcare Ltd.). The photographic films were then recorded using the UVI-prochemi gel documentation system (Uvitec, Cambridge, UK).

\section{P4/6H4 mAbs ratio evaluation and glycoform analysis}

The ratio between $\mathrm{P} 4$ and $6 \mathrm{H} 4 \mathrm{mAbs}$ was calculated by examining the films at equal time exposure, taking into account only the H-type sample signals. Samples were run three times and the films were analyzed using UVIprochemi software (Uvitec).

A glycoform analysis was performed to better determine whether the glycoform pattern of the three $\operatorname{PrP}^{\mathrm{Sc}}$ of the different BSE strains was detected by both extraction protocols. The samples were examined in three runs to evaluate the variability of the methods. Signals detected with $\mathrm{mAb} 6 \mathrm{H} 4$ were examined using UVI-prochemi software (Uvitec) and the values obtained for the di-glycosylated, mono-glycosylated and un-glycosylated bands were plotted as a graph.

\section{Acknowledgements}

The authors thank Sharon Lund, Kevin Hassal, Deborah Clouser and Caterina Lucia Florio, Paola Gazzuola, Sabrina Nodari, Antonio Longo for their excellent technical assistance. This research was funded by the ARS Specific Cooperative Agreement No. 58-3625-4-F217 "Study of atypical BSE".

\section{Author details}

${ }^{1}$ CEA, Istituto Zooprofilattico Sperimentale del Piemonte, Liguria e Valle d'Aosta, Via Bologna 148, 10154, Turin, Italy. ${ }^{2}$ United States Department of Agriculture, Animal and Plant Health Inspection Service (APHIS), National Veterinary Services Laboratories (NVSL), Pathobiology Laboratory, 1920 Dayton Ave, Ames, IA, 50010, USA. ${ }^{3}$ Virus and Prion Research Unit, National Animal Disease Center, United States Department of Agriculture, Agricultural Research Service (ARS), 1920 Dayton Ave, Ames, IA, 50010, USA. ${ }^{4}$ M. D. Anderson Cancer Center, Department of Veterinary Medicine and Surgery Unit 63, 1515 Holcombe Boulevard, Room TB.4055C, Houston, TX 770304009, USA. ${ }^{5}$ K224B Mosier Hall College of Veterinary Medicine, Kansas State University, Manhattan, KS 66506-5601, USA.

\section{Authors' contributions \\ JAR, MC, CC ${ }^{\#}$ and PA conceived of the study. JAR, SMH, CC $C^{\ddagger}, C C^{\#}, F M, C P$ and $A N H$ participated in its design. JAR, SMH, CC $C^{¥}, F M, C P, A Z P$ coordinated the study. ANH kindly provided the TME infected cattle tissue as positive control. CP and CML performed the immunohistochemical investigations, FM and JAR carried out the Western blot analyses. JAR and CC \# helped to draft the manuscript. CP and FM wrote the manuscript. CML, SMH, JJG, JAR, $C C^{\#}, C C^{¥}, \mathrm{BI}, \mathrm{PA}$ and FM contributed to manuscript revision. All authors have read and approved the final manuscript.}

\section{Competing interests}

The authors declare that they have no competing interests.
Received: 20 October 2010 Accepted: 29 September 2011

Published: 29 September 2011

\section{References}

1. Prusiner SB: Novel proteinaceus infectious particles cause scrapie. Science 1982, 216:136-144

2. Wells GAH, Scott AC, Johnson CT, Gunning RF, Hancock RD, Jeffrey M, Dawson $M$, Bradley R: A novel progressive spongiform encephalopathy in cattle. Vet Rec 1987, 121:419-420.

3. Number of cases of bovine spongiform encephalopathy (BSE) reported in the United Kingdom. [http://www.oie.int/animal-health-in-the-world/bsespecific-data/number-of-cases-in-the-united-kingdom/].

4. Number of reported cases of bovine spongiform encephalopathy (BSE) in farmed cattle worldwide (excluding the United Kingdom). [http:// www.oie.int/animal-health-in-the-world/bse-specific-data/number-ofreported-cases-worldwide-excluding-the-united-kingdom/].

5. Bruce ME, Will RG, Ironside JW, McConnell I, Drummond D, Suttie A, McCardle L, Chree A, Hope J, Birkett C, Cousens S, Fraser H, Bostock CJ: Transmission to mice indicate that "new variant" CJD is caused by the BSE agent. Nature 1997, 389:498-501.

6. Hill AF, Desbruslais M, Joiner S, Sidle KC, Gowland I, Collinge J, Doey L, Lantos P: The same prion strain causes VCJD and BSE. Nature 1997, 389:448-450.

7. Foster JD, Hope J, Fraser H: Transmision of bovine spongiform encephalopathy to sheep and goats. Vet Rec 1993, 133:339-341.

8. Eloit M, Adjou K, Coulpier M, Fontaine JJ, Hamel R, Lilin T, Messiaen S, Andreoletti O, Baron T, Bencsik A, Biacabe AJ, Beringue V, Laude H, Le Dur A, Vilotte JL, Comoy E, Deslys JP, Grassi J, Simon S, Lantier F, Sarradin P: BSE agent signatures in a goat. Vet Rec 2005, 156:523-524.

9. Wells GA, McGill IS: Recently described scrapie-like encephalopathies of animals: case definitions. Res Vet Sci 1992, 53:1-10

10. Pearson GR, Wyatt JM, Gruffydd-Jones TJ, Hope J, Chong A, Higgins RJ, Scott AC, Wells GA: Feline spongiform encephalopathy: fibril and PrP studies. Vet Rec 1992, 131:307-310.

11. Wilesmith JW, Wells GA, Cranwell MP, Ryan JB: Bovine spongiform encephalopathy: epidemiological studies. Vet Rec 1988, 123:638-644.

12. Wilesmith JW, Ryan JB, Atkinson MJ: Bovine spongiform encephalopathy: epidemiological studies on the origin. Vet Rec 1991, 128:199-203.

13. Eddy RG: Origin of BSE. Vet Rec 1995, 137:648.

14. Brown P, MCShane LM, Zanusso G, Detwiler L: On the question of sporadic or atypical bovine spongiform encephalopathy and Creutzfeldt-Jacob disease. Emerg Infect Dis 2006, 12:1816-1821.

15. Nicholson EM, Brunelle BW, Richt JA, Kehrli ME Jr, Greenlee JJ: Identification of a heritable polymorphism in bovine PRNP associated with genetic transmissible spongiform encephalopathy: evidence of heritable BSE. PIOS ONE 2008, 3:e2912.

16. Richt JA, Hall SM: BSE case associated with prion protein gene mutation. PLOS Pathog 2008, 4:e 1000156.

17. Simmons MM, Harris P, Jeffrey M, Meek SC, Blamire IW, Wells GA: BSE in Great Britain: consistency of the neurohistopathological findings in two randon annual samples of clinically suspect cases. Vet Rec 1996, 138:175-177.

18. Orge L, Simas JP, Fernades AC, Ramos M, Galo A: Similarity of the lesion profile of BSE in Portuguese cattle to that described in British cattle. Vet Rec 2000, 147:486-488.

19. Debeer S, Baron T, Bencsik A: Neuropathological characterization of French bovine spongiform encephalopathy cases. Histochem Cell biol 2003, 120:513-521.

20. Casalone C, Caramelli M, Crescio MI, Spencer YI, Simmons MM: BSE immunohistochemical patterns in the brainstem: a comparison between UK and Italian cases. Acta Neuropathol 2006, 111:444-449.

21. Jacobs JG, Langeveld JPM, Biacabe A-G, Acutis PL, Polak MP, GavierWiden D, Buschmann A, Caramelli M, Casalone C, Mazza M, Groschup M, Erkens JHF, Davidse A, van Zijderveld FG, Baron T: Molecular discrimination of atypical bovine spongiform encephalopathy strains from a geographical region spanning a wide area in Europe. J Clin Microbiol 2007, 45:1821-1829.

22. Wells GAH, Simmons MM: The essential lesion profile of bovine spongiform encephalopathy (BSE) in cattle is unaffected by breed or route of infection. Neuropathol Appl Neurobiol 1996, 22:453. 
23. Bruce ME, Boyle A, Cousens S, McConnell I, Foster J, Goldmann W, Fraser H: Strain characterization of natural sheep scrapie and comparison with BSE. J Gen Virol 2002, 83:695-704.

24. Green R, Horrocks C, Wilkinson A, Hawkins SA, Ryder SJ: Primary isolation of the bovine spongiform encephalopathy agent in mice: agent definition based on a review of 150 transmissions. J Comp Path 2005, 132:117-131.

25. Casalone C, Zanusso G, Acutis PL, Ferrari S, Capucci L, Tagliavini F, Monaco S, Caramelli M: Identification of a second bovine amyloidotic spongiform encephalopathy: molecular similarities with sporadic Creutzfeldt-Jacob disease. Proc Natl Acad Sci USA 2004, 101:3065-3070.

26. Wells GAH, Wilesmith JW, McGill IS: Bovine spongiform encephalopathy: a neuropathological perspective. Brain Pathol 1991, 1:69-78.

27. Biacabe $A G$, Laplanche $J$, Ryder $S$, Baron T: Distinct molecular phenotypes in bovine prion diseases. EMBO Rep 2004, 5:110-115.

28. Harmeyer S, Pfaff E, Groschup MH: Synthetic peptide vaccines yield monoclonal antibodies to cellular and pathological prion proteins of ruminants. J Gen Virol 1998, 79:937-945.

29. Polak MP, Zmudzinski JF, Jacobs JG, Langeveld JPM: Atypical status of bovine spongiform encephalopathy in Poland: a molecular typing study. Arch Virol 2008, 153:69-79.

30. Buschmann A, Gretzschel A, Biacabe A-G, Schiebel K, Corona C, Hoffmann C, Eiden M, Baron T, Casalone C, Groschup MH: Atypical BSE in Germany - Proof of transmissibility and biochemical characterization. Vet Microbiol 2006, 117:103-116.

31. Richt JA, Kunkle RA, Alt D, Nicholson EM, Hamir AN, Czub S, Kluge J, Davis AJ, Hall SM: Identification and characterization of two bovine spongiform encephalopathy cases diagnosed in the United States. J Vet Diagn Invest 2007, 19:142-154.

32. González L, Martin S, Begara-McGorum I, Hunter N, Houston F, Simmons M, Jeffrey M: Effects of agent strain and host genotype on PrP accumulation in the brain of sheep naturally and experimentally affected with scrapie. J Comp Path 2002, 126:17-29.

33. Baron T, Biacabe AG: Origin of bovine spongiform encephalopathy. Lancet 2006, 367:297-298.

34. Biacabe AG, Morignat E, Vulin J, Calavas D, Baron T: Atypical bovine spongiform encephalopathies, France, 2001-2007. Emerg Infect Dis 2008, 14:298-300.

35. European Commission: Regulation (EC) No 162/2009 of the European Parliament and of the Council of 26 February 2009 amending Annexes III and X to Regulation (EC) No 999/2001 laying down rules for the prevention and control and eradication of certain transmissible spongiform encephalopathies. Off J Eur Communities Volume 55 European Commission. Official Journal of European Communities 2009, 11-16.

36. Lombardi G, Casalone C, D'Angelo A, Gelmetti D, Torcoli G, Barbieri I, Corona C, Fasoli E, Farinazzo A, Fiorini M, Gelati M, lulini B, Tagliavini F, Ferrari S, Caramelli M, Monaco S, Capucci L, Zanusso G: Intraspecies trasmission of BASE induces clinical dullness and amyotrophic changes. PLOS Pathog 2008, 4:e1000075.

37. Gavier-Widén D, Nöremark M, Langeveld JPM, Stack M, Biacabe A-J, Vulin J, Chaplin M, Richt JA, Jacobs J, Acín C, Monleón E, Renström L, Klingeborn B, Baron TGM: Bovine spongiform encephalopathy in Sweden: an H-type variant. J Vet Diagn Invest 2008, 20:2-10.

38. Linder $E$, Miettinen $A$ : Prozone effects in indirect immunofluorescence. Scand J Immunol 1976, 5:513-519.

39. Everbroeck BV, Pals $P$, Martin JJ, Cras P: Antigen retrieval in prion protein immunohistochemistry. J Histochem Cytochem 1999, 47:1465-1467.

40. O'Rourke KI, Baszler TV, Besser TE, Miller JM, Cutlip RC, Wells GA, Ryder SJ, Parish SM, Hamir AN, Cockett NE, Jenny A, Knowles DP: Preclinical diagnosis of scrapie by immunohistochemistry of third eyelid lymphoid tissue. J Clin Microbiol 2000, 38:3254-9.

41. Manual of Diagnostic Tests and Vaccines for Terrestrial Animals 2011. Chapter 2.4.6. Bovine Spongiform Encephalopathy (version adopted in May 2010). [http://www.oie.int/fileadmin/Home/eng/Health_standards/ tahm/2.04.06_BSE.pdf].

42. Bozzetta E, Acutis PL, Martucci F, Nappi R, Casalone C, Mazza M, Caramelli M: Evaluation of rapid tests for the diagnosis of transmissible spongiform encephalopathies in sheep and goats. Acta Neuropathol 2004, 107:559-562.

43. Schaller O, Fatzer R, Stack MJ, Clark J, Cooley W, Biffiger K, Egli S, Doherr M, Vandevelde M, Heim D, Oesh B, Moser M: Validation of a Western immunoblotting procedure for bovine $\mathrm{PrP}^{\mathrm{Sc}}$ detection and its use as a rapid surveillance method for the diagnosis of bovine spongiform encephalopathy (BSE). Acta Neuropathol 1999, 98:437-443.

doi:10.1186/1756-0500-4-376

Cite this article as: Porcario et al:: Evaluation of two sets of immunohistochemical and Western blot confirmatory methods in the detection of typical and atypical BSE cases. BMC Research Notes 2011 4:376.

\section{Submit your next manuscript to BioMed Central and take full advantage of:}

- Convenient online submission

- Thorough peer review

- No space constraints or color figure charges

- Immediate publication on acceptance

- Inclusion in PubMed, CAS, Scopus and Google Scholar

- Research which is freely available for redistribution

Submit your manuscript at www.biomedcentral.com/submit
Biomed Central 